\title{
Systemic mastocytosis presenting with a prominent B lymphocyte proliferation in the bone marrow and extensive fibrosis of the spleen
}

\author{
Nalan Neşe ${ }^{\mathrm{a}, *}$, Seçkin Çağırgan ${ }^{\mathrm{b}}$, Yeşim Ertan $^{\mathrm{c}}$, Ayhan Sönmez $^{\mathrm{b}}$, Saliha Soydan ${ }^{\mathrm{c}}$ and Mine Hekimgil ${ }^{\mathrm{c}}$ \\ ${ }^{a}$ Department of Pathology, Celal Bayar University Faculty of Medicine, Manisa, Turkey \\ ${ }^{\mathrm{b}}$ Department of Hematology, Ege University Faculty of Medicine, Izmir, Turkey \\ ${ }^{\mathrm{c}}$ Department of Pathology, Ege University Faculty of Medicine, Izmir, Turkey
}

\begin{abstract}
Systemic mastocytosis is a disease characterized by multifocal mast cell proliferation in the bone marrow or other extracutaneous organs. Because of loosely scattered and hypo-/agranular mast cells, the diagnosis is sometimes very difficult. In the bone marrow, mast cell infiltration may be associated with prominent lymphoid infiltration leading to a misdiagnosis of a low grade non-Hodgkin lymphoma.

A 49-year-old woman presented with right arm and leg pain, psychiatric symptoms, and diarrhea for four years. Physical examination and laboratory investigation revealed hepatosplenomegaly, anemia, mild thrombocytosis, mild leucocytosis and lymphocytosis. In the bone marrow biopsy, there was a prominent B lymphocyte proliferation reminiscent of a low grade non-Hodgkin lymphoma/leukemia and there were some spindle cells aggregates in paratrabecular location. The consecutive bone marrow biopsies were similar to the first. The subsequent splenectomy specimen exhibited striking fibrosis. In the lymph node sections, there was marginal zone hyperplasia.

Multifocal accumulations of mast cells were strongly positive with mast cell tryptase and CD117 on immunohistochemical staining, though no metachromasia was identified in Giemsa and Toluidine Blue stained aspirates and tissue sections, probably due to hypo-/agranulation of mast cells.

The case was presented to emphasize the importance of the antibody to mast cell tryptase in the diagnosis of mastocytosis and to discuss problems of differential diagnosis of systemic mastocytosis.
\end{abstract}

Keywords: Systemic mastocytosis, fibrosis of spleen, mast cell tryptase, smoldering variant

\section{Introduction}

The term "mastocytosis" is used to describe a heterogeneous group of disorders characterized by abnormal growth and accumulation of mast cells (MCs) [1-4]. Mostly the disease is localized and confined to the skin,

* Corresponding author: Nalan Neşe. Postal address: Celal Bayar University Faculty of Medicine, Department of Pathology, Manisa, Turkey 45010. Tel.: +90 5332433133; +90 2362323133 (302); Fax: +90 236 23701213; E-mail: nalannese@ hotmail.com. and this form is classified as cutaneous mastocytosis. Systemic mastocytosis (SM) is best defined as a multifocal proliferation of abnormal tissue MCs, preferentially involving the bone marrow, skin, spleen, liver, gastrointestinal tract, and lymph nodes. SM is a persistent disease, now accepted as a myeloproliferative disorder, accounting for only $15-20 \%$ of all cases [3, 5]. SM presents with an indolent or aggressive clinical course and may cause considerable diagnostic difficulties. Clinically, various symptoms may be seen such as urticaria, pruritis, abdominal pain, cramping, diarrhea, 
weight loss, bone pain, headache, and symptoms associated with organ dysfunctions [6]. Histopathologically, it is usually diagnosed by the examination of bone marrow, the site most frequently affected by SM [2-5].

In most cases of SM, bone marrow MCs show atypia, like cytoplasmic extensions forming fusiform shapes, oval nuclei with eccentric position, bi- or multilobed nuclei and hypogranulated cytoplasm. In a small group of cases, the abnormal morphology of MCs gives negative results in Toluidine Blue staining [7]. In these cases, mast cell tryptase is a very useful immunohistochemical marker in the diagnosis of SM.

SM may be associated with myeloid disorders including myeloproliferative diseases, myelodysplastic syndromes, acute myeloid leukemia, and very rarely non-Hodgkin lymphoma, often low grade subtypes, in less than $5 \%$ to $20 \%$ of cases [3-6,8,9]. Furthermore, in the bone marrow biopsies of cases with SM, admixture of reactive $\mathrm{T}$ and $\mathrm{B}$ lymphocytic infiltration may accompany MCs $[6,10]$.

\section{Case}

The case is a 49-year-old woman presenting arm and leg pain, psychiatric symptoms, vomiting and diarrhea for four years. Physical examination revealed hepatomegaly and splenomegaly. She did not have any skin lesions at the time of presentation. Laboratory findings revealed anemia, mild thrombocytosis, mild leukocytosis and lymphocytosis (41\%). Radiological examination (magnetic resonance imaging and scintigraphy) showed increase in bone density and findings recommended a metabolic bone disease.

In the first bone marrow biopsy, there was a mild to prominent interstitial and focal lymphocytic infiltration in a hypercellular background predominantly consisting of CD20-positive B-cells (Fig. 1A, 1B), mimicking infiltration of a low grade B-cell non-Hodgkin lymphoma. The B-cell lymphocytic infiltration did not express CD5, CD10, CD23, CD43, and cyclinD1. In addition to lymphoid cells, there were plump oval or spindle-shaped mast cells resembling fibroblasts, loosely scattered, interstitial or peritrabecular and vascular in distribution (Fig. 1C). These cells with hypogranular or agranular large cytoplasms and oval or lobulated nuclei did not show any metachromasia in Toluidine Blue staining. In the hypocellular bone marrow aspirates, there were only a few large mast cells with basophilic agranular or hypogranular cytoplasm and bland cytology on Giemsa staining. Increased mar- row fibrosis was detected with reticulin stain (grade II). The histological evaluation of the bone revealed an osteopetrosis-like osteopathy characterized with broad, sclerotic bone trabecules.

A year after presentation, hepatomegaly and splenomegaly progressed and weight loss $(6 \mathrm{~kg})$ was identified. Peripheral blood counts were similar except a steady increase in leukocytes. Second and third bone marrow biopsies were performed at two months and one year after presentation, respectively, revealing similar histopathological and immunohistochemical findings. During $14^{\text {th }}$ month of follow-up, splenectomy and excisional biopsy of mesenteric lymph node were carried out. On gross examination, the spleen weighed $480 \mathrm{gr}$, the capsule was thickened, and cut surface was fibrotic. The microscopic examination revealed striking fibrosis producing a diffuse interlacing pattern with loss of normal splenic architecture (Fig. 1E). Spindle-shaped mast cells were detected within the thick fibrous bands surrounding atrophic areas of white and red pulp. Paracortical and marginal zone hyperplasia was identified in mesenteric lymph node sections. Scattered spindle cells were seen in the subcapsular and interfollicular areas.

Follow-up of the patient did not reveal any significant change in her physical and hematological condition. Two years and four months after the presentation, she complained of pruritis, abdominal cramps, diarrhea, and urticaria localized to the legs. The radiological examination showed sclerosis and increased density of the bones of pelvis, wrist, and cruris. The last bone marrow biopsy demonstrated a compact dense, sharply demarcated, multifocal infiltration of atypical, spindleshaped or plump polygonal cells. The re-evaluation and immunohistochemical staining for MC tryptase of all previous specimens indicated a progressive increase in the density of MC infiltrates (Fig. 1D, 1F). In the most recent biopsy sample, focal aggregates consisting of at least 15 mast cells were identified and over $25 \%$ of the MCs exhibited clear spindling, fulfilling the criteria for SM. MCs were CD117 positive and CD2 negative.

\section{Discussion}

This interesting case of SM is presented and discussed here because of various problems of differential diagnosis, caused by prominent B lymphocyte proliferation in the bone marrow, striking fibrosis of the spleen and marginal zone hyperplasia in the mesenteric lymph node masking MC infiltrations in these tissues. 


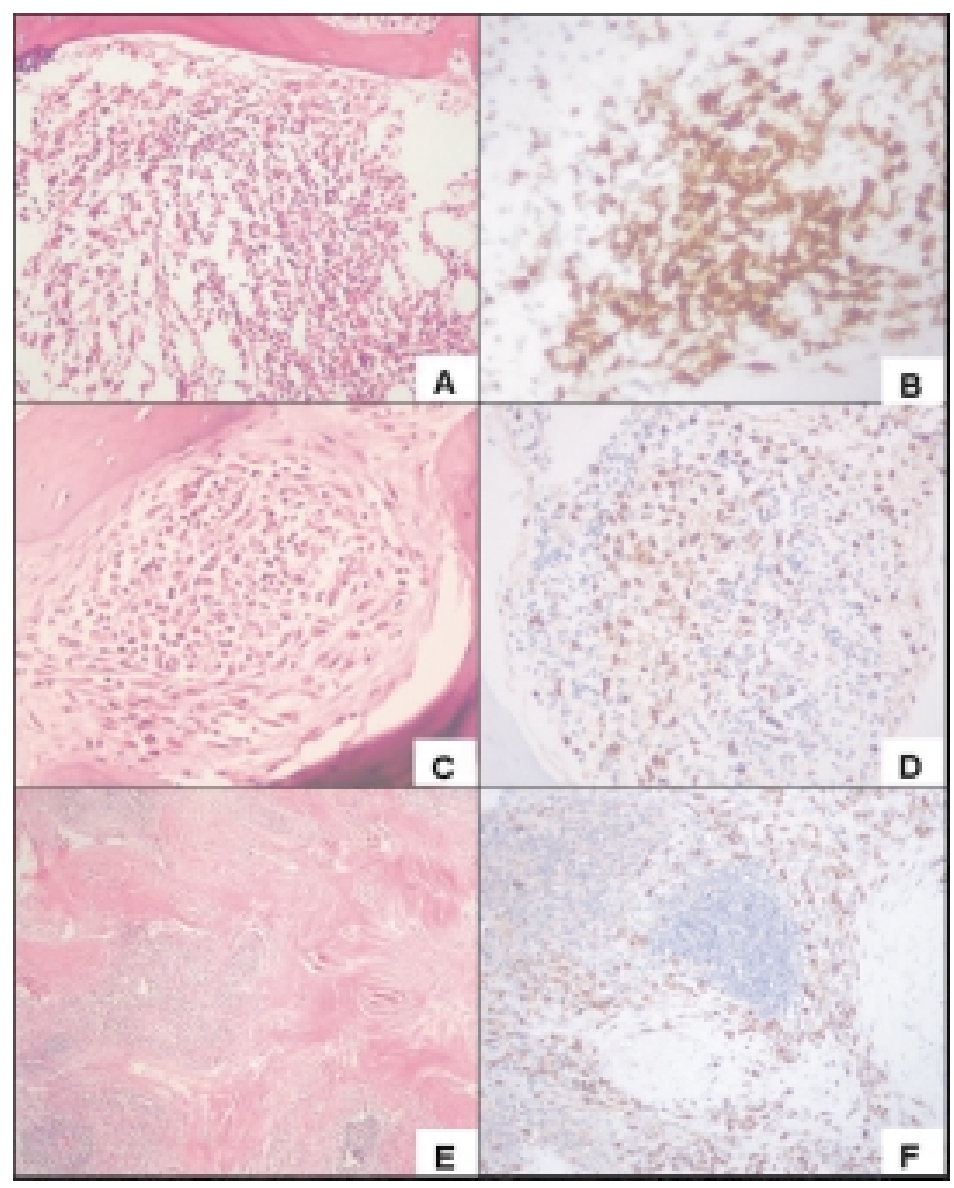

Fig. 1. A) Lymphocytic infiltration in the initial bone marrow biopsy (X100). B) CD20 staining of lymphoid cells in the bone marrow (X100). C) Focal aggregates of plump oval or spindle-shaped cells resembling fibroblasts in the initial bone marrow biopsy (X200). D) MC tryptase staining of the initial bone marrow biopsy (X200). E) Striking fibrozis of the spleen (X40). F) Spindle cells in splenic fibrotic areas stained with MC tryptase (X40).

According to the recently updated WHO classification of mastocytosis [4] proposed by Valent et al. [3], the diagnosis of SM is primarily based on the number of MCs in aggregates ( $>15 \mathrm{MCs}$ ) and the density of MC infiltrates with abnormal morphology ( $>25 \%)$ in the bone marrow and/or other extracutaneous tissues, presence of c-kit mutations, aberrant expression of $\mathrm{CD} 2$ and/or CD25, and serum total tryptase values $>20 \mathrm{ng} / \mathrm{ml}[5,11]$. The diagnosis of SM may be delayed due to many causes as presented in this case $[2,3$, $8,12]$. Clinically, signs and symptoms are diverse and may simulate some hematological disorders. Histologically, the infiltration is often focal and dense, especially in the forms that show a malignant course, the MCs are morphologically abnormal and lack metachromatic granules $[5,7,13]$. Another reason of misdiagnose, is that the underlying histological picture of the bone marrow might be complicated by secondary hematological disorders $[3,4,6,8-10,12]$. Moreover, it is known that the infiltrates are not composed exclusively of MCs, but also contain eosinophils and lymphocytes [10]. Bone marrow infiltrates may be pure mastocytic, mixed mastocytic/lymphocytic or predominantly lymphocytic. The immunohistochemical and molecular studies revealed that the lymphoid infiltration associated with SM is an admixture of T and B lymphocytes or predominantly T-cells and is polyclonal and reactive in nature $[6,10]$. However in cases with a predominant Bcell infiltration, a diagnosis of low-grade non-Hodgkin lymphoma is often suspected initially, as in our case [6, 12].

Immunostaining with antibodies against mast cell tryptase, has proved to be of great value in establishing the correct diagnosis, especially in cases of malignant mastocytosis and it is always superior to metachromatic stains, since these atypical MCs are often very im- 
mature and hypogranulated $[2-5,7,12,13]$. To avoid underestimation of MC infiltration and to classify the mast cell diseases, the degree of infiltration and/or the percentage of MCs should always be assessed by antimast cell tryptase immunostaining [12].

Fibrosis is also commonly associated with SM, further complicating the picture [3,12]. MCs are known to be the source of fibrogenic cytokines. The percentage of MCs in Giemsa-stained bone marrow smears is generally low due to dense fibrosis, even if the biopsies present a high infiltration grade, rendering difficulties in diagnosis.

Because MCs are virtually absent in both normal and hyperplastic splenic tissue, the presence of even a few loosely scattered MCs may be indicative of primary MC disease [12]. We believe that tryptase immunohistochemistry should be performed on all splenectomy materials presenting a dense fibrosis with interlacing pattern. Since MCs may be present in relatively large numbers in chronic lymphadenitis, mainly within the sinusoids, it is often very difficult to confirm or exclude lymph node involvement by SM [12].

Osteosclerosis and osteopetrosis-like osteopathy are frequently seen in indolent forms of SM, but apparently not in aggressive forms such as mast cell leukemia [2,6]. Examination of bone trabecules might draw attention to the underlying systemic disease.

Aberrant expression of CD2 and CD25 in the flow cytometric evaluation of the bone marrow and demonstration of codon 816 mutations of $c$-kit are very useful in differentiating the clonal nature of the MCs from reactive MCs that may accompany various conditions [3$5,9,11,13]$. In our case, flow cytometry could not to be done due to dry tap and immunohistochemical staining was performed in which $\mathrm{CD} 117$ was strongly positive, but $\mathrm{CD} 2$ was negative. Nevertheless, CD2 is reported as negative or restricted to a subpopulation of neoplastic MCs on immunohistochemical evaluation [13].

We presented a case with a delayed diagnose of SM and discussed the pitfalls of diagnosis such as diverse clinical symptoms; findings preventing to identify MCs in the bone marrow biopsies like pronounced increase in reticulin fibres, prominent lymphoid proliferation, and lack of metachromatic granules on Toluidine Blue staining; extensive fibrosis in the spleen; and marginal zone hyperplasia in the mesenteric lymph node. The diagnosis was relatively straightforward in the last bone marrow biopsy with identification of the pathognomonic compact dense peritrabecular and -vascular aggregates of atypical MCs. We believe that the patient has presented smouldering SM, the provisional sub- variant of indolent SM, because of the long-lasting unrecognized SM without any skin lesions or impaired organ function due to infiltration by neoplastic MCs. Since this provisional subvariant could be a prephase of aggressive SM or SM with associated clonal hematologic non-MC lineage disease, these cases should be followed-up with multiple bone marrow biopsies. In similar cases, more frequent use of adjunctive immunohistochemical stains, such as MC tryptase, is necessary to reach a diagnosis.

\section{References}

[1] P. Valent, C. Akin, W.R. Sperr, H.P. Horny and D.D. Metcalfe, Mast cell proliferative disorders: current view on variants recognized by the World Health Organization, Hematol Oncol Clin North Am 17(5) (2003), 1227-1241.

[2] H.P. Horny, C. Sillaber, D. Menke, E. Kaiserling, M. Mehrmann, B. Stehberger et al., Diagnostic value of immunostaining for tryptase in patients with mastocytosis, Am J Surg Pathol 22(9) (1998), 1132-1140.

[3] P. Valent, H.P. Horny, L. Escribano, B.J. Longley, C.Y. Li, L.B. Schwartz et al., Diagnostic criteria and classification of mastocytosis: a consensus proposal, Leuk Res 25(7) (2001), 603-625.

[4] P. Valent, H.P. Horny, C.Y. Li, J.B. Longley, D.D. Metcalfe, R.M. Parwaresch et al., Mastocytosis (Mast cell disease). World Health Organization (WHO) Classification of Tumours. Pathology \& Genetics, in: Tumours of Haematopoietic and Lymphoid Tissues, (Vol. 1), E.S. Jaffe, N.L. Harris, H. Stein and J.W. Vardiman, eds, 2001, pp. 291-302.

[5] P. Valent, C. Akin, W.R. Sperr, M. Mayerhofer, M. Fodinger, R. Fritsche-Polanz et al., Mastocytosis: pathology, genetics, and current options for therapy, Leuk Lymphoma 46(1) (Jan. 2005), 35-48.

[6] H.P. Horny, K. Lange, K. Sotlar and P. Valent, Increase of bone marrow lymphocytes in systemic mastocytosis: reactive lymphocytosis or malignant lymphoma? Immunohistochemical and molecular findings on routinely processed bone marrow biopsy specimens, J Clin Pathol 56(8) (2003), 575-578.

[7] E.C. Stevens and N.S. Rosenthal, Bone marrow mast cell morphologic features and hematopoietic dyspoiesis in systemic mast cell disease, Am J Clin Pathol 116(2) (2001), 177-182.

[8] J.H. Butterfield and C.Y. Li, Bone marrow biopsies for the diagnosis of systemic mastocytosis: is one biopsy sufficient? Am J Clin Pathol 121(2) (2004), 264-267.

[9] H.P. Horny, K. Sotlar, W.R. Sperr and P. Valent, Systemic mastocytosis with associated clonal haematological non-mast cell lineage diseases: a histopathological challenge, J Clin Pathol 57(6) (2004), 604-608.

[10] H.P. Horny and E. Kaiserling, Lymphoid cells and tissue MCs of BM lesions in SM: a histological and immunohistological study, Br J Haematol 69(4) (1988), 449-455.

[11] J.H. Jordan, S. Walchshofer, W. Jurecka, I. Mosberger, W.R. Sperr, K. Wolff et al., Immunohistochemical properties of bone marrow mast cells in systemic mastocytosis: evidence for expression of CD2, CD117/Kit, and bcl-x (L), Hum Pathol 32(5) (2001), 545-552.

[12] H.P. Horny and P. Valent, Diagnosis of mastocytosis: general histopathological aspects, morphological criteria, and immunohistochemical findings, Leuk Res 25(7) (2001), 543-551. 
[13] M. Krokowski, K. Sotlar, M.T. Krauth, M. Födinger, P. Valent and H.P. Horny, Delineation of patterns of bone marrow mast cell infiltration in systemic mastocytosis. Value of CD25, cor- relation with subvariants of the disease, and separation from mast cell hyperplasia, Am J Clin Pathol 124(4) (2005), 560568. 


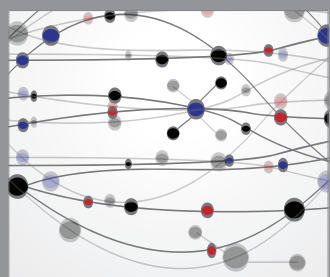

The Scientific World Journal
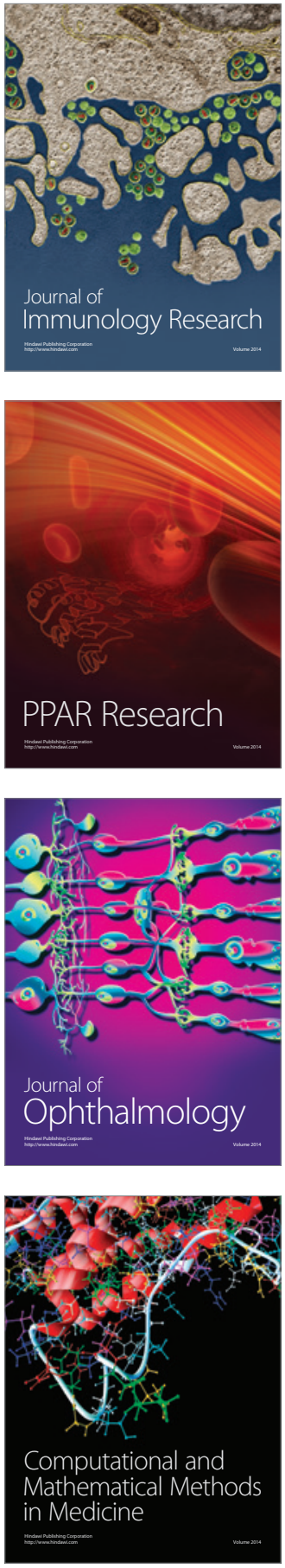

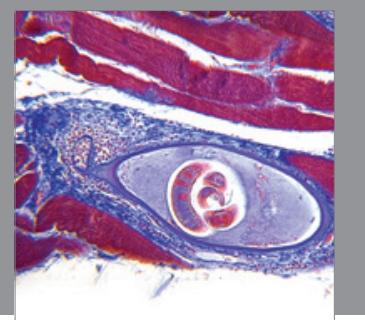

Gastroenterology

Research and Practice
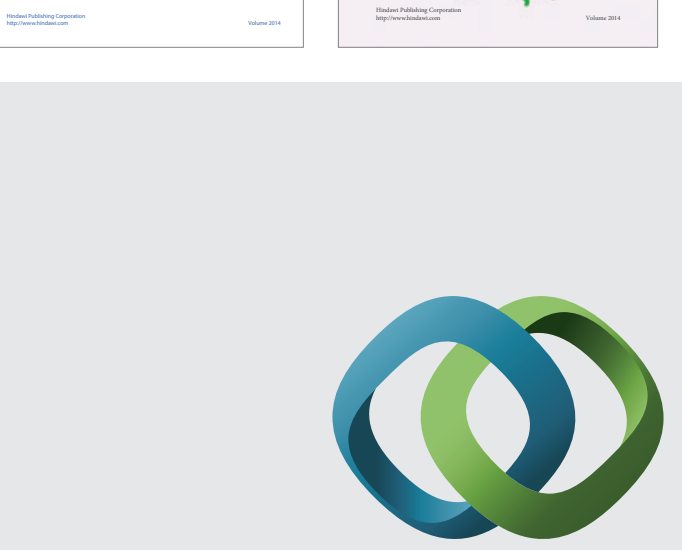

\section{Hindawi}

Submit your manuscripts at

http://www.hindawi.com
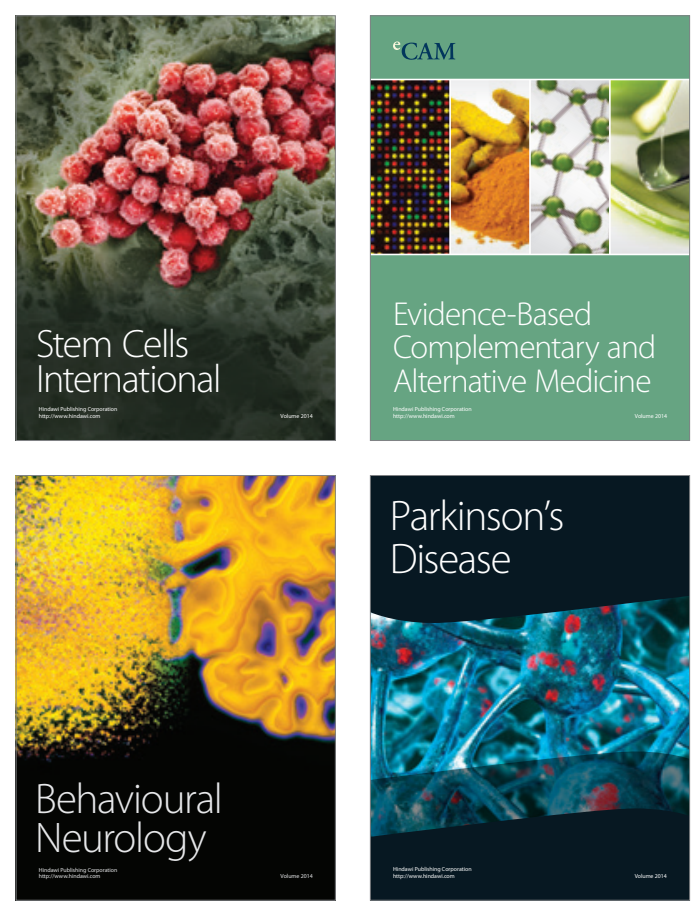

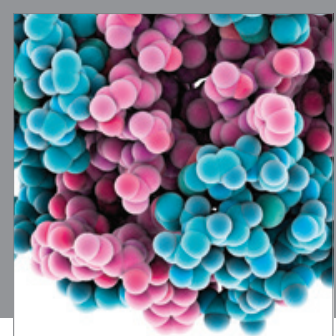

Journal of
Diabetes Research

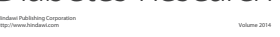

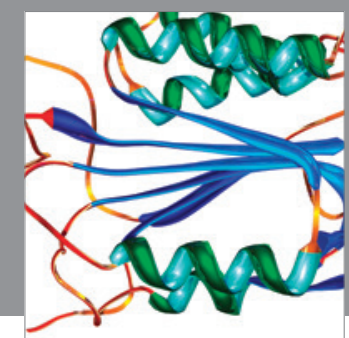

Disease Markers
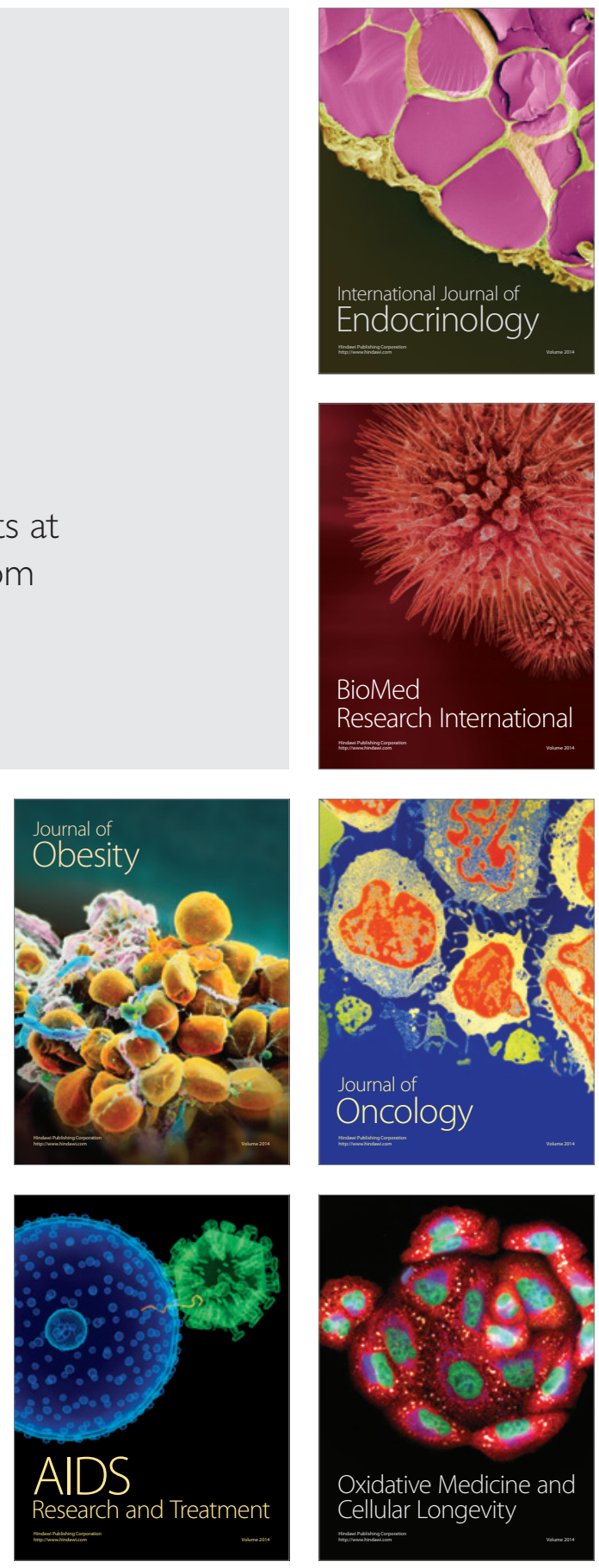